This item was submitted to Loughborough's Research Repository by the author.

Items in Figshare are protected by copyright, with all rights reserved, unless otherwise indicated.

\title{
Kinetic equation for nonlinear resonant wave-particle interaction
}

PLEASE CITE THE PUBLISHED VERSION

http://dx.doi.org/10.1063/1.4962526

\section{PUBLISHER}

(c) The Authors. Published by AIP Publishing

\section{VERSION}

VoR (Version of Record)

\section{PUBLISHER STATEMENT}

This work is made available according to the conditions of the Creative Commons Attribution-NonCommercialNoDerivatives 4.0 International (CC BY-NC-ND 4.0) licence. Full details of this licence are available at: https://creativecommons.org/licenses/by-nc-nd/4.0/

\section{LICENCE}

CC BY-NC-ND 4.0

\section{REPOSITORY RECORD}

Artemyev, A.V., Anatoly Neishtadt, Alexei Vasiliev, and D. Mourenas. 2019. "Kinetic Equation for Nonlinear Resonant Wave-particle Interaction". figshare. https://hdl.handle.net/2134/22601. 


\section{Kinetic equation for nonlinear resonant wave-particle interaction}

A. V. Artemyev, A. I. Neishtadt, A. A. Vasiliev, and D. Mourenas

Citation: Physics of Plasmas 23, 090701 (2016); doi: 10.1063/1.4962526

View online: http://dx.doi.org/10.1063/1.4962526

View Table of Contents: http://aip.scitation.org/toc/php/23/9

Published by the American Institute of Physics

\section{Articles you may be interested in}

Probability of relativistic electron trapping by parallel and oblique whistler-mode waves in Earth's radiation belts Physics of Plasmas 22, 112903 (2015); 10.1063/1.4935842

Non-diffusive resonant acceleration of electrons in the radiation belts

Physics of Plasmas 19, 122901 (2012); 10.1063/1.4769726

Transverse $\mathrm{eV}$ ion heating by random electric field fluctuations in the plasmasphere

Physics of Plasmas 24, 022903 (2017); 10.1063/1.4976713

Nonlinear electron acceleration by oblique whistler waves: Landau resonance vs. cyclotron resonance Physics of Plasmas 20, 122901 (2013); 10.1063/1.4836595

Modelling nonlinear electrostatic oscillations in plasmas

Physics of Plasmas 23, 122103 (2016); 10.1063/1.4968520

Electron holes in phase space: What they are and why they matter

Physics of Plasmas 24, 055601 (2017); 10.1063/1.4976854

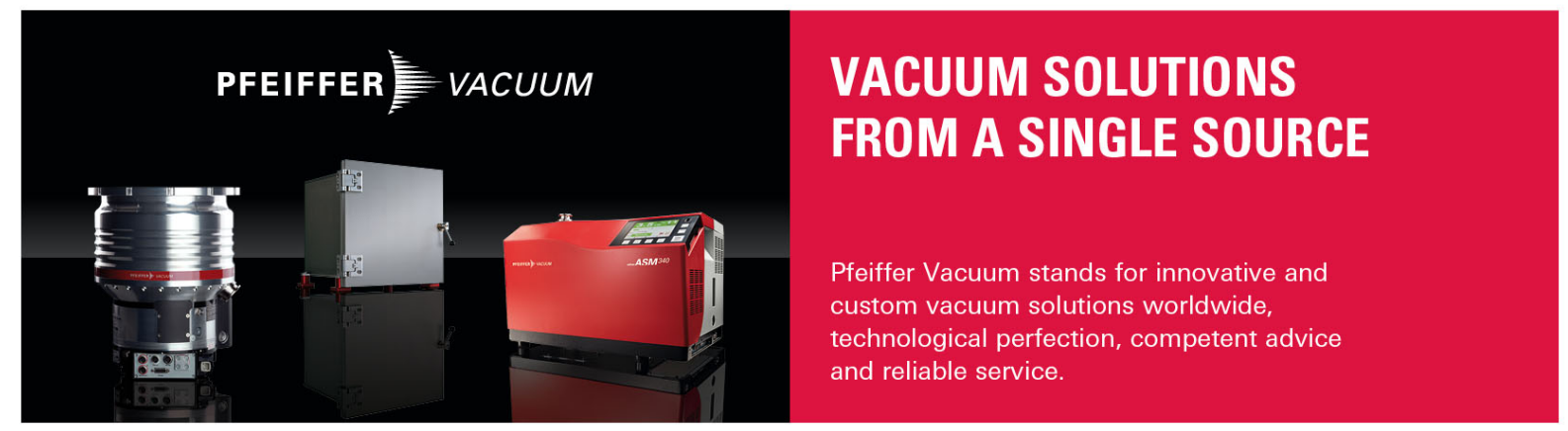




\title{
Kinetic equation for nonlinear resonant wave-particle interaction
}

\author{
A. V. Artemyev, ${ }^{1,2, a)}$ A. I. Neishtadt, ${ }^{2,3}$ A. A. Vasiliev, ${ }^{2}$ and D. Mourenas ${ }^{4}$ \\ ${ }^{1}$ Institute of Geophysics and Planetary Physics, UCLA, Los Angeles, California 90095-1567, USA \\ ${ }^{2}$ Space Research Institute, RAS, Moscow, Russia \\ ${ }^{3}$ Department of Mathematical Sciences, Loughborough University, Loughborough LE11 3TU, \\ United Kingdom \\ ${ }^{4} C E A, D A M, D I F$, Arpajon, France
}

(Received 29 June 2016; accepted 29 August 2016; published online 8 September 2016)

\begin{abstract}
We investigate the nonlinear resonant wave-particle interactions including the effects of particle (phase) trapping, detrapping, and scattering by high-amplitude coherent waves. After deriving the relationship between probability of trapping and velocity of particle drift induced by nonlinear scattering (phase bunching), we substitute this relation and other characteristic equations of waveparticle interaction into a kinetic equation for the particle distribution function. The final equation has the form of a Fokker-Planck equation with peculiar advection and collision terms. This equation fully describes the evolution of particle momentum distribution due to particle diffusion, nonlinear drift, and fast transport in phase-space via trapping. Solutions of the obtained kinetic equation are compared with results of test particle simulations. Published by AIP Publishing.

[http://dx.doi.org/10.1063/1.4962526]
\end{abstract}

Resonant interactions of a particle ensemble with a broad spectrum of low amplitude electromagnetic waves are conventionally described by the diffusion (Fokker-Planck) equation of the quasi-linear theory. ${ }^{9,22}$ The timescale of the resonant wave-particle interactions is then defined by the inverse of the finite wave-packet dispersion rate. ${ }^{12,17}$ In inhomogeneous plasmas (or background magnetic field), an additional stochastization of the resonant charged particle motion introduces a new timescale of the wave-particle interactions ${ }^{19,20}$ and justifies the applicability of the quasi-linear diffusion equation even for a very narrow wave spectrum. ${ }^{1}$ One of the most successful examples of application of the quasi-linear theory is the very accurate description of relativistic electron acceleration and scattering in the Earth's radiation belts. However, when the wave amplitudes become sufficiently high, the applicability of the quasi-linear approach breaks down ${ }^{17}$ and the nonlinear effects such as particle trapping and nonlinear scattering (phase bunching) $)^{11,15}$ start to play an important role. These effects are well studied and described analytically for test particle trajectories (e.g., Refs. 2, 3, 6, and 18), but the problem of their proper inclusion into a Fokker-Planck equation for a full description of the long-term evolution of a particle ensemble has, so far, eluded solution (e.g., see discussion in Ref. 16).

If the wave field energy is much smaller than the particle kinetic energy, the wave-particle interactions are described by the Hamiltonian $H=H_{0}+\varepsilon H_{1} \cos \phi$ where the unperturbed Hamiltonian $H_{0}$ describes particle motion in the background magnetic field, and the wave-induced perturbation $\varepsilon H_{1} \cos \phi$ depends on the wave phase $\phi$ (where the weakness of perturbations is taken into account via the small parameter $\varepsilon \ll 1$ ). Unperturbed motion consists of several types of oscillations (gyrorotation around the background magnetic field, bounce oscillations between magnetic mirror points,

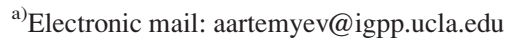

etc., e.g., Ref. 20). If one of these oscillations turns out to be faster than the wave phase variation (which rate $\dot{\phi}$ is about the wave frequency $\omega$ ), the perturbation should be expanded in harmonics of this oscillation $H_{1} \cos \phi=\sum_{n} H_{1}^{(n)} \cos \phi^{(n)}$, where each harmonic can be considered separately (later we omit index $n){ }^{20}$ The phase $\phi$ varies much faster than other variables, and we assume for simplicity that its rate $\dot{\phi} \sim 1 / \varepsilon$ (in the general case, $\dot{\phi}$ is a large parameter independent of $1 / \varepsilon$, but for most important cases, the temporal variation of the perturbation $\partial\left(H-H_{0}\right) / \partial t \sim \varepsilon \dot{\phi}$ should be about $O(1)$ to significantly affect particle motion). It is convenient to introduce $\phi$ as a new variable and to consider the pair of conjugate variables $(\phi, I)$, where $I$ is the particle normalized momentum. ${ }^{2,3,18}$ Changing the timescale $t \rightarrow t \varepsilon$, we obtain a system where phase changes at a rate $\sim 1$, whereas other variables (momenta and coordinates) evolve over the timescale $\tau=t \varepsilon$. To parameterize the dependence of $H$ on these slow variables in terms of dependence on $\tau$, we consider a simple but rather general $1 \frac{1}{2}$ degrees of freedom system with Hamiltonian $H=H_{0}(\tau, I)+\varepsilon H_{1}(\tau, I) \cos \phi$. The resonant condition $\dot{\phi}=\partial H_{0} / \partial I=0$ has a solution $I=I_{R}(\tau)$. Expansion around $I_{R}$ gives the following Hamiltonian:

$$
H=\Lambda+\frac{1}{2} g\left(I-I_{R}\right)^{2}+\varepsilon B \cos \phi,
$$

where $\quad \Lambda(\tau)=H_{0}\left(\tau, I_{R}(\tau)\right), g(\tau)=\left(\partial^{2} H_{0} / \partial I^{2}\right)_{I=I_{R}}, B(\tau)$ $=H_{1}\left(\tau, I_{R}(\tau)\right)$. We introduce the new variable $P_{\phi}=\left(I-I_{R}\right)$ and rewrite the new Hamiltonian $\mathcal{F}$ of perturbations $H-\Lambda$ in a form

$$
\mathcal{F}=\frac{1}{2} g P_{\phi}^{2}+\varepsilon A \phi+\varepsilon B \cos \phi,
$$

where $\varepsilon A=\partial I_{R} / \partial t$ and $\left(\phi, P_{\phi}\right)$ are conjugate variables. The general Hamiltonian (2) describes resonant systems with fast phase (see a general derivation of this Hamiltonian in 
Ref. 14). The corresponding Hamiltonian equations $\dot{P}_{\phi}=$ $-\varepsilon A+\varepsilon B \sin \phi, \dot{\phi}=g P_{\phi}$ describe the nonlinear pendulum with a torque that appears in the majority of systems describing wave-particle resonant interactions (see, e.g., reviews $2-4$, and 18 and references therein). The analysis of the Hamiltonian (2) will provide the needed characteristics of particle scattering and acceleration by the waves. Below we consider only sufficiently intense coherent waves such that $B>|A|$.

For $a(\tau)=B /|A|>1$ (for definiteness, we consider $B>0$ and $g>0$ ), the phase portrait of Hamiltonian (2) contains a region filled with closed trajectories. The existence of such a region guarantees the nonlinear character of waveparticle interaction (see more details in Refs. 2, 3, and 18). Particles moving along closed trajectories oscillate around the resonance $\dot{\phi}=0$ and are called trapped particles. Particles moving along open trajectories are transient and are scattered by the wave. If the area $S$ of the trapped region grows, some transient particles can get trapped into resonance with the wave. $S$ is given by equation (see Eq. (2)) $S(\tau)=\oint P_{\phi} d \phi=\sqrt{8 \varepsilon B / g} s(a)$ where

$$
s(a)=\int_{\phi_{-}}^{\phi_{+}} \sqrt{a^{-1}\left(\phi_{+}-\phi\right)+\cos \phi_{+}-\cos \phi} d \phi,
$$

and $\phi_{-}$is a root of equation $a \sin \phi=-1$, whereas $\phi_{+}$is a root of equation $\left(\phi_{-}-\phi\right)+a\left(\cos \phi_{-}-\cos \phi\right)=0$. The relative number of particles which can be trapped by waves during one passage through resonance is called the probability of trapping $\Pi$ and is defined as a ratio of $d S / d t$ and phase flux through the resonance ${ }^{3,14}$

$$
\Pi=\frac{d S}{d t} /\left|\int_{0}^{2 \pi} \dot{P}_{\phi} d \phi\right|=\frac{1}{2 \pi|A|} \frac{d S}{d \tau},
$$

where $\dot{P}_{\phi}$ is defined by Hamiltonian equations for (2), and $\Pi=0$ if $d S / d \tau<0$. Both $S$ and $\Pi$ are small variables $\sim \sqrt{\varepsilon}$. Once being trapped, particles move in resonance with the wave until the area $S(\tau)$ returns to its value at the moment of trapping $\tau_{\text {trap }}$ (see scheme in Fig. 1). During the time interval of trapped motion, particle momentum $I$ varies as $I_{R}$, and particles escape from resonance with larger (or smaller) $I$ (or larger (smaller) energy $H(I, \tau)$ ). This effect corresponds to particle acceleration via trapping. 2,3,16,18 Since the duration of trapping acceleration can be rather long and variations of $I$ can be large (both being independent of $\varepsilon$ ), this process cannot be described as a local diffusion.

Transient particles passing through the resonance without being trapped are scattered with a change of momentum $I$ given by equation $\Delta I=\int \dot{I} d t=\sqrt{2 \varepsilon B / g} h\left(\phi^{*}, a\right)$, where

$$
h\left(\phi^{*}, a\right)=\int_{-\infty}^{\phi *} \frac{\operatorname{sign}(A) \sqrt{a} \sin \phi d \phi}{\sqrt{\phi^{*}-\phi+a\left(\cos \phi^{*}-\cos \phi\right)}},
$$

and $\phi^{*}$ is defined by the particle energy $\mathcal{F}^{*}=\varepsilon|A|\left(\phi^{*}\right.$ $\left.+a \cos \phi^{*}\right)$ taken at $P_{\phi}=0$. The integral (5) depends on $\tau$ and $\theta=\left(\phi^{*}+a \cos \phi^{*}\right) / 2 \pi$. The function $h(\theta)$ is periodic

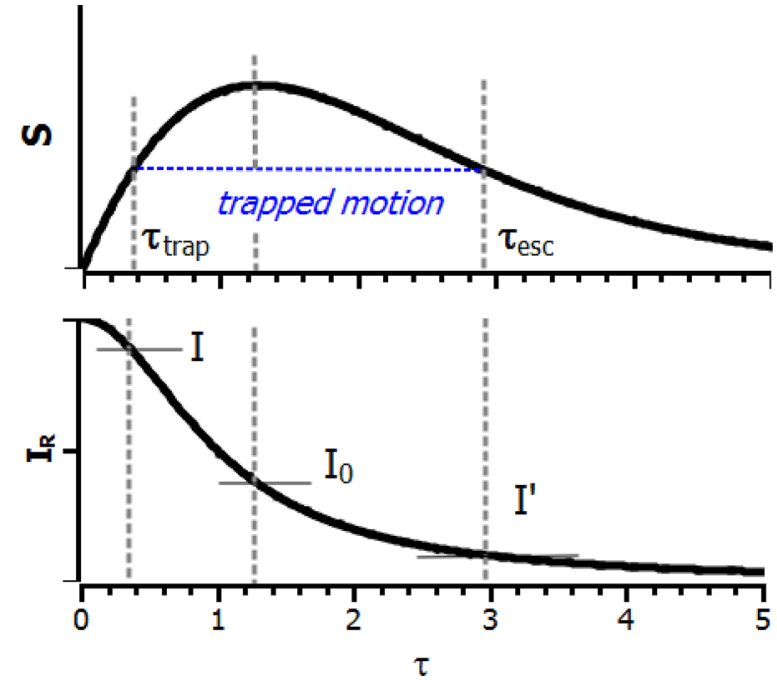

FIG. 1. A schematic view of area $S(\tau)$ and resonant momentum $I_{R}(\tau)$ profiles. A particle becomes trapped by the wave at $\tau=\tau_{\text {trap }}$, when the area $S$ grows and $I=I_{R}$. During trapped motion, momentum varies as $I_{R}(\tau)$. When the area $S$ returns back to the value $S\left(\tau_{\text {trap }}\right)$, the trapped particle escapes from resonance, at $\tau=\tau_{\text {esc }}$. After escape, the particle has a momentum $I_{R}\left(\tau_{\text {esc }}\right)=I^{\prime}$. For simplicity, we consider here systems with a single maximum of $S$ at $I=I_{0}$.

(see, e.g., Ref. 3), whereas the mean value $\langle h\rangle_{\theta}=$ $-\operatorname{sign}(A) s(a) / \pi$ for $a>1$ and $\langle h\rangle_{\theta}=0$ for $a<1$ (see Ref. 14). For wave-particle interactions in a magnetic field, averaging over initial conditions (gyrophases) of resonant particles is equivalent to an averaging over $\theta$. This allows us to derive two expressions describing the evolution of the $\theta$-averaged particle distribution $f(I)$ under the influence of nonlinear and quasi-linear scattering: ${ }^{8}$ the velocity of drift $V=\langle\Delta I\rangle / \tau_{0}=-\operatorname{sign}(A) S /\left(2 \pi \tau_{0}\right)$ and the diffusion coefficient $D=\operatorname{Var}(\Delta I) / \tau_{0}=2 \varepsilon(B / g) \operatorname{Var}(h)$, where $\tau_{0}$ is the time interval between two successive passages through the resonance and Var is the variance. This expression for the diffusion coefficient $D$ coincides with the formula derived previously for a narrow wave spectrum in the frame of the quasi-linear theory. ${ }^{1}$ Coefficients $\Pi(\tau), V(\tau), D(\tau)$ can be rewritten as $\Pi(I), V(I), D(I)$ because $I=I_{R}$ at resonance.

We have derived expressions for $\Pi(I), V(I)$, and $D(I)$. These general characteristics of nonlinear wave-particle resonant interaction have been derived analytically and tested numerically many times for different plasma systems. ${ }^{1-3,16,18}$ However, to our best knowledge, no equation providing the relationship between $\Pi$ and $V(I)$ has been yet available. Hereafter, we shall derive this relationship and use it to construct (for the first time) a generalized Fokker-Planck equation which will include all effects described by $\Pi(I), V(I)$, and $D(I)$. Using the definition $\varepsilon A=\partial I_{R} / \partial t$ and Eq. (4), we obtain

$$
\frac{d V}{d I}=-\frac{\operatorname{sign}(A)}{2 \pi \tau_{0}} \frac{d S}{d t}\left(\frac{d I}{d t}\right)_{I=I_{R}}^{-1}=-\frac{1}{2 \pi \varepsilon|A| \tau_{0}} \frac{d S}{d t}=-\frac{\Pi}{\tau_{0}} .
$$

Using the above-defined $D, V$, and $\Pi / \tau_{0}$ and considering a trapping/escape event as a rapid change of particle $I$ to $I^{\prime}$ (where $I^{\prime}$ is defined by equations $I^{\prime}=I_{R}\left(\tau_{\text {esc }}\right), S\left(\tau_{\text {trap }}\right)$ $=S\left(\tau_{\text {esc }}\right)$, see Fig. 1), we can now write a Fokker-Planck equation describing the full evolution of the distribution 
function $f(I)$ under the influence of both scattering and trapping (the diffusion term being written in its divergent form)

$$
\begin{aligned}
\frac{\partial f}{\partial t}= & \frac{\partial}{\partial I}\left(D \frac{\partial f}{\partial I}\right)-\frac{\partial(V f)}{\partial I} \\
& +\int_{0}^{\infty}\left(f(\tilde{I}) Q_{\tilde{I} \rightarrow I}-f(I) Q_{I \rightarrow \tilde{I}}\right) d \tilde{I},
\end{aligned}
$$

where particle transport $I^{\prime} \rightarrow I$ via trapping is defined by probability $Q_{I^{\prime} \rightarrow I}$ and particle departure from $I$ by probability $Q_{I \rightarrow I^{\prime}}$. Let us consider $S(\tau)$ rewritten as $S\left(I_{R}\right)$ with monotonous $I_{R}(\tau)$ and assume for simplicity that $S$ has only one maximum at $I_{R}=I_{0}$ (see Fig. 1). At resonance $I=I_{R}$, and thus $S=S(I)$. For $I>I_{0}$ we have $S$ growing and a finite probability of trapping $Q_{I \rightarrow I^{\prime}}=\left(\Pi(I) / \tau_{0}\right) \delta\left(I-I^{\prime}\right)$. For $I<I_{0}$, the area $S$ decreases and particles escape from resonance. The corresponding function is $Q_{I^{\prime} \rightarrow I}=\left(\Pi\left(I^{\prime}\right) / \tau_{0}\right) \delta\left(I-w\left(I^{\prime}\right)\right)$, where function $w\left(I^{\prime}\right)$ gives the value $I^{\prime}>I_{0}$ that particles should have when getting trapped to escape later from resonance with $I<I_{0}$ ( $w$ is defined by the $S(I)$ profile, see Fig. 1 ), and $\Pi\left(I^{\prime}\right)$ is the probability of particle trapping at $I^{\prime}>I_{0}$. The integral operator in Eq. (7) can then be re-formulated as $-f(I) \Pi(I) / \tau_{0}$ for $I>I_{0}$ and $f\left(I^{\prime}\right) \Pi\left(I^{\prime}\right)\left|d w / d I^{\prime}\right|^{-1} / \tau_{0}=$ $(d V / d I) f\left(I^{\prime}\right)$ for $I<I_{0}$, where we used

$$
\frac{d w\left(I^{\prime}\right)}{d I^{\prime}}=\frac{d I}{d I^{\prime}}=\frac{d V\left(I^{\prime}\right) / d I^{\prime}}{d V(I) / d I}=-\frac{\Pi\left(I^{\prime}\right) / \tau_{0}}{d V(I) / d I},
$$

and $V\left(I^{\prime}\right)=V(I)$ because $S\left(\tau_{\text {trap }}\right)=S\left(\tau_{\text {esc }}\right)$. Using these expressions and Eq. (6), we can rewrite Eq. (7) in its final form

$$
\frac{\partial f}{\partial t}=\frac{\partial}{\partial I}\left(D \frac{\partial f}{\partial I}\right)-V \frac{\partial f}{\partial I}-\frac{d V}{d I}\left(f-f\left(I^{\prime}\right)\right) \Theta(I),
$$

where $I=w\left(I^{\prime}\right), \Theta(I)=1(0)$ for $I>I_{0}\left(I<I_{0}\right)$. Equation (8) is a Fokker-Planck equation generalized to include effects of nonlinear scattering (drift $V$ ) and fast transport in phase space (nonlocal term $\sim f(I)-f\left(I^{\prime}\right)$ ). The timescale of diffusion is $\varepsilon^{-1}$ (since $D \sim \varepsilon$ ), whereas the timescales of drift and trapping-induced transport are much shorter $\sim \varepsilon^{-1 / 2}$. For systems with small wave amplitudes $(a<1), V$ and $d V / d I$ vanish and Eq. (8) reduces to the usual quasi-linear diffusion equation.

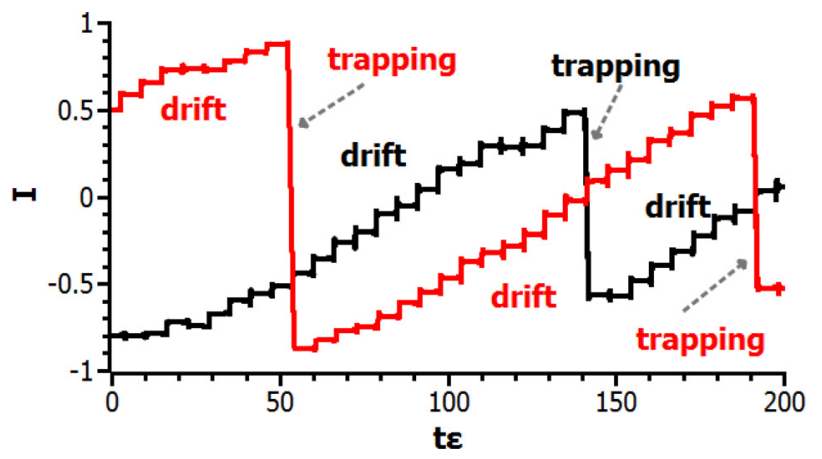

FIG. 2. Two test trajectories (shown by black and red lines) for Hamiltonian (1).
To check Eq. (8), we compare its solution with results of numerical trajectory integration for the initial system (1). We consider a simple example of Hamiltonian (1) with $\Lambda=0$, $g=1, I_{R}=\sin \tau$, and $B(\tau)$ a periodic function of $\tau$ (period is $\tau_{0}=2 \pi / \varepsilon$ ) with a single maximum over $\tau \in\left[\tau_{0} / 4,3 \tau_{0} / 4\right]$ and equals to zero for $\tau \in\left[0, \tau_{0} / 4\right],\left[3 \tau_{0} / 4, \tau_{0}\right]$. The resonant momentum $I_{R}$ varies from -1 to 1 and particles with all $I \in$ $[-1,1]$ pass through the resonance over one period $\tau_{0}$. Figure 2 shows two examples of test trajectories calculated over a long time interval. Particles can be trapped at a positive $I$ and transported to negative $I$ (in this system $I_{0}=0$ ), whereas nonlinear drift $V$ transports particles back from negative $I$ to positive $I$. Both processes of trapping and drift coexist with diffusive scattering. Particle behavior shown in Fig. 2 is a typical behavior in plasma systems with nonlinear wave-particle interactions (see, e.g., trajectories in Refs. 3 and 16).

For our test system, we calculate $\Pi$ from Eq. (4), $\tau_{0} V=$ $-\sqrt{8 \varepsilon B} s(a) / 2 \pi$ from Eq. (3), diffusion coefficient $D$, and function $w(I)$, and express them as functions of $I=I_{R}$. To check the analytical formulas for $\Pi, V$, and $D$, we integrate numerically $10^{7}$ trajectories with different initial $I$ and calculate changes $\Delta I$ over one period $\tau_{0}$ (i.e., for a single resonant interaction). The ratio of the number of particles which were trapped into resonance over the total number of particles gives the numerical probability of trapping $\Pi$. For untrapped particles, we calculate the average value of $\Delta I$ and its variance $\operatorname{Var}(\Delta I)$. These two functions, depending on the initial $I$, are then used to calculate the numerical $V$ and $D$. Figure 3 demonstrates that our analytical expressions provide quite accurate descriptions of the trapping probability, drift velocity, and diffusion coefficient.
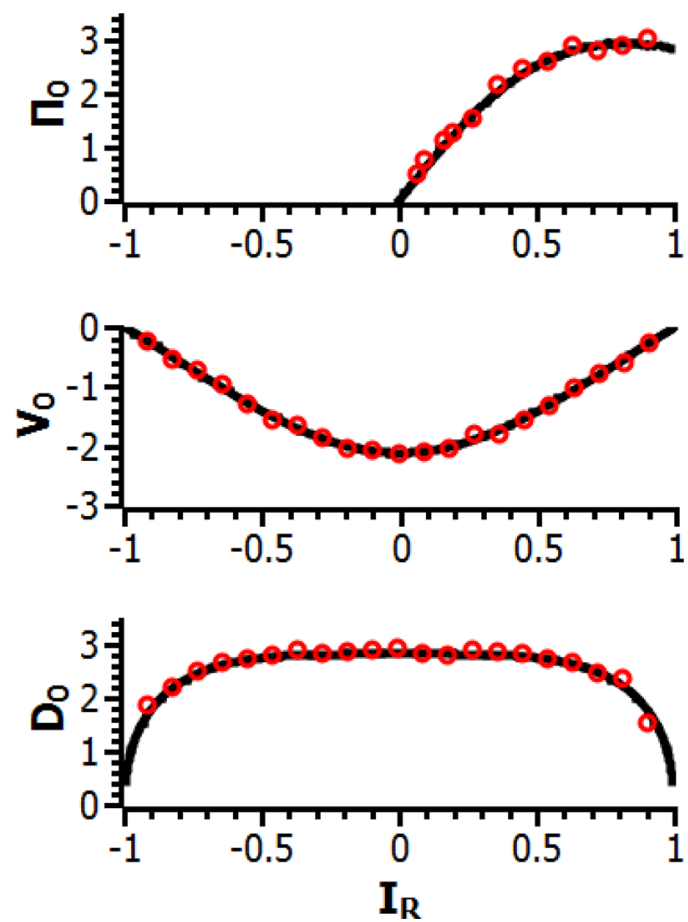

FIG. 3. Comparisons of analytical expressions for $\Pi_{0}=\Pi \tau_{0} / \sqrt{\varepsilon}, V_{0}=V \tau_{0} / \sqrt{\varepsilon}$, and $D_{0}=D \tau_{0} / \varepsilon$ (black curves) with numerical results (red circles). 

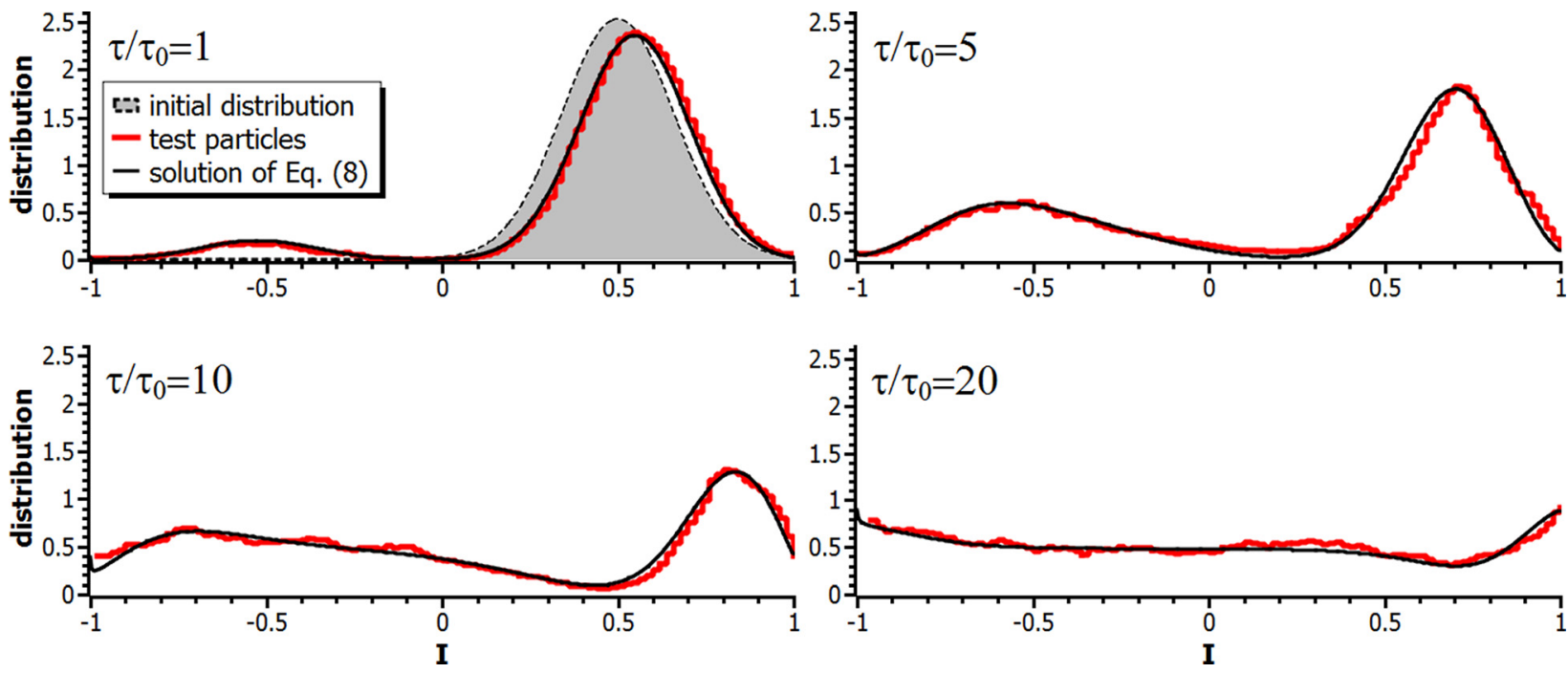

FIG. 4. Distribution function $f(I)$ at four moments of time. The initial distribution is displayed in grey, black curves show the numerical solution of the new generalized Fokker-Planck equation (8), and red curves are obtained from direct numerical integration (with the explicit Runge-Kutta method) of equations of motion for Hamiltonian system (1) with $10^{7}$ different values of initial $(I, \phi)$. For numerical integration of Eq. (8), we use a tridiagonal matrix algorithm (implicit method), whereas the last term of Eq. (8) is calculated with $f$ from the previous time step.

The above analytical coefficients $V$ and $D$ have been substituted into Eq. (8), which was solved numerically for a particular initial distribution $f_{0}(I)$. This solution is compared in Figure 4 with the results of direct numerical integration of $10^{7}$ test particle trajectories for Hamiltonian (1). All three expected effects of trapping and scattering are recovered: (1) fast transport of trapped particles from the initial distribution peak at positive $I$ toward negative $I$; (2) particle nonlinear drift toward positive $I$; and (3) a slower diffusive flattening.

Based on the successful comparisons displayed in Fig. 4, we can conclude that Eq. (8) accurately describes all the effects of nonlinear as well as quasi-linear wave-particle interactions. This new equation can therefore be considered as a generalization of the classical Fokker-Planck equation derived in the restricted frame of quasi-linear theory. Moreover, its practical, analytical form allows a much faster resolution than with particle simulations, while retaining all the complex and entangled variations. Using the same proposed approach, a more general 2D form of Eq. (8) could be derived, allowing swift calculations of the evolution of particle energy and pitch-angle distributions in many plasma systems where wave intensity and coherency are sufficient to drive nonlinear interactions, as in the Earth's radiation belts ${ }^{2,3}$ and magnetized laboratory plasmas, ${ }^{7,21}$ inertial confinement fusion, ${ }^{4,10}$ or Penning-Malmberg traps. ${ }^{5,13}$

The work of A.V.A., A.A.V., and A.I.N was supported by the Russian Scientific Fund, Project No. 14-12-00824.
${ }^{1}$ J. M. Albert, J. Geophys. Res. 115, A00F05, doi:10.1029/2009JA014732 (2010).

${ }^{2}$ J. M. Albert, X. Tao, J. Bortnik, Y. Omura, D. Nunn, and D. Summers, Dynamics of the Earth's Radiation Belts and Inner Magnetosphere (American Geophysical Union, 2013), pp. 243-264.

${ }^{3}$ A. Artemyev, O. Agapitov, D. Mourenas, V. Krasnoselskikh, V. Shastun, and F. Mozer, Space Sci. Rev. 200, 261 (2016).

${ }^{4}$ D. Benisti and L. Gremillet, Phys. Rev. E 91, 042915 (2015).

${ }^{5}$ R. C. Davidson, Physics of Nonneutral Plasmas, 2nd ed. (Imperial College Press/World Scientific Publishing, London, UK, 2001).

${ }^{6}$ A. G. Demekhov, V. Y. Trakhtengerts, M. Rycroft, and D. Nunn, Geomagn. Aeron. 49, 24, doi:10.1134/S0016793209010034 (2009).

${ }^{7}$ I. Y. Dodin, P. F. Schmit, J. Rocks, and N. J. Fisch, Phys. Rev. Lett. 110, 215006 (2013)

${ }^{8}$ D. Dolgopyat, Repulsion from Resonances, Memoires de la Société Mathématique de France Vol. 128 (Société Mathématique de France, 2012).

${ }^{9}$ W. E. Drummond and D. Pines, Nucl. Fusion Suppl. 3, 1049 (1962).

${ }^{10}$ K. Hara, T. Chapman, J. W. Banks, S. Brunner, I. Joseph, R. L. Berger, and I. D. Boyd, Phys. Plasmas 22, 022104 (2015).

${ }^{11}$ V. I. Karpman and D. R. Shklyar, Sov. JETP 35, 500 (1972).

${ }^{12}$ C. F. Kennel and F. Engelmann, Phys. Fluids 9, 2377 (1966).

${ }^{13}$ J. H. Malmberg and J. S. deGrassie, Phys. Rev. Lett. 35, 577 (1975).

${ }^{14}$ A. I. Neishtadt, Hamiltonian Systems with Three or More Degrees of Freedom, NATO ASI Series C 533 (Kluwer, Dordrecht, 1999), p. 193.

${ }^{15}$ D. Nunn, J. Plasma Phys. 6, 291 (1971).

${ }^{16}$ Y. Omura, Y. Miyashita, M. Yoshikawa, D. Summers, M. Hikishima, Y. Ebihara, and Y. Kubota, J. Geophys. Res. 120, 9545, doi:10.1002/ 2015JA021563 (2015).

${ }^{17}$ V. D. Shapiro and R. Z. Sagdeev, Phys. Rep. 283, 49-71 (1997).

${ }^{18}$ D. Shklyar and H. Matsumoto, Sur. Geophys. 30, 55 (2009).

${ }^{19}$ V. V. Solovev and D. R. Shkliar, Sov. JETP 63, 272-277 (1986).

${ }^{20} \mathrm{~V}$. Y. Trakhtengerts and M. J. Rycroft, Whistler and Alfvén Mode Cyclotron Masers in Space (Cambridge University Press, 2008).

${ }^{21}$ B. Van Compernolle, X. An, J. Bortnik, R. M. Thorne, P. Pribyl, and W. Gekelman, Phys. Rev. Lett. 114, 245002 (2015).

${ }^{22}$ A. A. Vedenov, E. Velikhov, and R. Sagdeev, Nucl. Fusion Suppl. 2, 465 (1962). 